\title{
Medial Meniscal Tears in Anterior Cruciate Ligament- Deficient Knees: Effects of Posterior Tibial Slope on Medial Meniscal Tear
}

\author{
Jae-Jeong Lee, MD, Yun-Jin Choi, MD, Keun-Young Shin, MD and Chong-Hyuk Choi, MD, PhD \\ Department of Orthopedic Surgery, Gangnam Severance Hospital, Yonsei University College of Medicine, Seoul, Korea
}

Purpose: To evaluate the incidence of meniscal tears in patients with chronic anterior cruciate ligament (ACL) -deficient knees, and to determine the influence of posterior tibial slope (PTS) on medial meniscal tears in ACL-deficient knees.

Materials and Methods: We reviewed 174 patients (174 knees) with a mean age of 30.7 years who underwent ACL reconstruction for chronic ACL tears. We divided the patients into two groups: low group ( 135 knees with a PTS $\left.<13^{\circ}\right)$ and high group $\left(39\right.$ knees with a PTS $\left.\geq 13^{\circ}\right)$.

Results: The incidence of medial meniscus tears was 44\% (77/174), and that of lateral meniscus tears was 35\% (61/174). The mean PTS in patients with medial meniscal tears was $11.4^{\circ} \pm 3.0^{\circ}$, whereas that in patients without medial meniscal tears was $9.8^{\circ} \pm 2.4^{\circ}$. The incidence of meniscal tears was $57.8 \%(78 / 135)$ in the low group and $89.7 \%(35 / 39)$ in the high group $(\mathrm{p}<0.001)$.

Conclusions: Our data demonstrate that PTS $\geq 13^{\circ}$ is a risk factor for secondary medial meniscal tears in ACL-deficient knees. So, we suggest that PTS is one of the considerations for determining early ACL reconstruction to prevent secondary meniscal tears.

Key words: Chronic anterior cruciate ligament deficient knee, Medial meniscal tear, Posterior tibial slope.

\section{Introduction}

The meniscus is frequently damaged in anterior cruciate ligament (ACL) injuries or in the degenerative process over a period of several years ${ }^{1)}$. The predominance of lateral meniscal tears has been demonstrated with acute ACL rupture, whereas the incidence of medial meniscal tears significantly increases with chronic ACL insufficiency ${ }^{2}$. The lateral meniscus is relatively loosely attached to the tibial plateau, which allows it to be quite

Received July 3, 2011; Revised (1st) August 12, 2011;

(2nd) October 10, 2011; Accepted October 23, 2011.

Correspondence to: Chong-Hyuk Choi, MD, PhD.

Department of Orthopedic Surgery, Gangnam Severance Hospital,

Yonsei University College of Medicine,

146-92 Dogok-dong, Gangnam-gu, Seoul 135-720, Korea.

Tel: +82-2-2019-3415, Fax: +82-2-573-5393

Email: choi8422@yuhs.ac

This is an Open Access article distributed under the terms of the Creative Common Attribution Non-Commercial License (http://creativecommons.org/licenses/by-nc/3.0/) which permits unrestricted non-commercial use, distribution, and reproduction in any medium, provided the original work is properly cited.

Copyright ๑ 2011. KOREAN KNEE SOCIETY

www.jksrr.org mobile, and performs little or no stabilizing function on the knee. The medial meniscus, however, is firmly attached to the tibia, especially at the posterior horn ${ }^{3,4)}$. This firm attachment allows the medial meniscus to serve as a knee stabilizer, and various studies have shown that it is a significant restraint to anterior tibial translation in ACL-deficient knees ${ }^{5-7)}$. Biomechanical studies have indicated that the meniscus performs this function by acting as a mechanical block or wedge ${ }^{8,9)}$. Their results suggest that the loss of ACL function increases anterior tibial translation, allowing the meniscus to engage the femoral condyle and act as a wedge against the tibia.

Radiographic studies have shown that there is a linear relationship between posterior tibial slope (PTS) and anterior tibial translation during weight bearing: the greater the PTS, the greater the anterior tibial translation, in both ACL-intact and ACL-deficient knees ${ }^{10,11)}$. Our hypothesis is that secondary medial meniscal tears occur more frequently in ACL deficient knees with greater PTS.

The objectives of this study were to evaluate the incidence of meniscal tears in patients with chronic ACL-deficient knees and to determine the influence of PTS on secondary medial meniscal tears in ACL-deficient knees. 


\section{Materials and Methods}

A retrospective review was performed on 202 patients who underwent ACL reconstruction by the senior author between January 2005 and May 2009. We excluded 28 patients who had multiple ligament injuries and/or reconstruction in the acute phase (within 3 months of injury). The remaining 174 patients (148 males and 26 females) were included in this cohort study. Their average age was 30.7 years (range, 14 to 53 years). The average time to surgery was 14.8 months (range, 3 to 140 months).

Although meniscus tears could be classified according to the location and type, we classified meniscus tears only according to the location. To classify the location of a tear on arthroscopy, each meniscus was considered to have three segments: the anterior horn, body, and posterior horn. In addition, the tears were divided into peripheral tears (those involving the red zone) and central tears (those involving the red-white zone and the white zone).

In order to measure the PTS, we used the tibial proximal anatomic axis (TPAA), which gives high reliability among the tibial axis ${ }^{12}$. On lateral radiographs, the TPAA was defined as a line connecting the two central points of the tibial cortex at $5 \mathrm{~cm}$ and $15 \mathrm{~cm}$ below the tibial tuberosity. On the lateral radiographs, the line connecting the highest anterior and posterior points of the medial plateau was regarded as a line for the tibial slope. The angle between the tangent line to the medial plateau and the perpendicular line to the TPAA was defined as PTS (Fig. 1). PTS

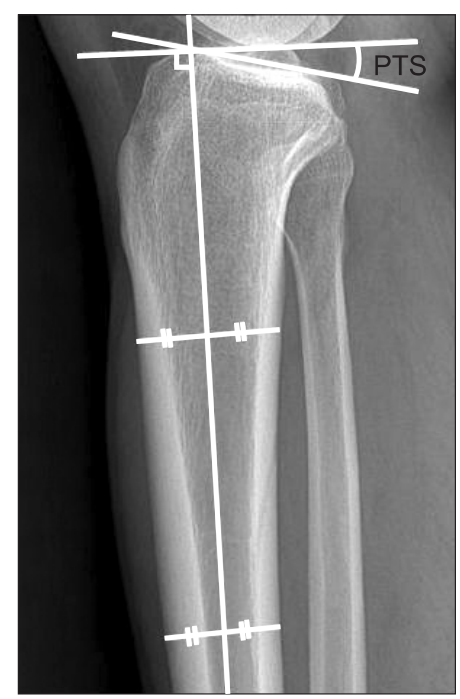

Fig. 1. The posterior tibial slope (PTS) was measured as the angle between a line tangent to the medial plateau and a line perpendicular to the tibial proximal anatomic axis. was independently measured by two orthopedic surgeons who were blinded to case status. The reliability of PTS measurements was assessed using the intraclass correlation coefficient. The interobserver reliability was 0.940 . The mean values of the measurements taken by the two surgeons were used in the assessment. The patients were divided into two groups according to their PTS. The average PTS measured on lateral radiographs is $10^{\circ} \pm 3^{\circ}$ in the literature, which was used as a reference for our study ${ }^{10)}$. In order to analyze the influence of PTS on secondary meniscal tears, we divided the patients into two groups: low group $\left(\mathrm{PTS}<13^{\circ}\right)$ and high group $\left(\mathrm{PTS} \geq 13^{\circ}\right)$.

The statistical analyses were performed using SAS (SAS Institute, Cary, NC, USA) software. Descriptive statistics including means, standard deviations, and minimum and maximum values were calculated for every group. Mann-Whitney U test was performed to detect significant effects of age, sex, body mass index (BMI) and the interval from injury to ACL reconstruction between the two groups. The chi square test was used to determine whether there were significant differences in tear pattern between two groups. If there were significant differences, a post-hoc test with the Bonferroni adjustment was used to determine which groups were significantly different from the others. The level of significance for all statistical tests was established at $\mathrm{p}<0.05$.

\section{Results}

The distribution of meniscal tears is presented in Tables 1-3. The overall incidence of meniscal injuries was 65\% (113/174) in the total chronic ACL-deficient knees. The incidence of medial meniscal tears was $44 \%(77 / 174)$, and that of lateral meniscal tears was $35 \%$ (61/174). The average PTS measured on the lateral radiographs was $10.5^{\circ} \pm 2.8^{\circ}$. The mean PTS in patients with medial meniscal tears was $11.4^{\circ} \pm 3.0^{\circ}$, whereas that in patients without medial meniscal tears was $9.8^{\circ} \pm 2.4^{\circ}$. In contrast, the mean PTS in patients with and without lateral meniscal tears was $10.6^{\circ} \pm 2.9^{\circ}$ and $10.5^{\circ} \pm 2.8^{\circ}$, respectively.

Between the low group and high group, no significant

Table 1. The Radial Zone Distribution of Medial Meniscal Tears in Anterior Cruciate Ligament-Tear Zone Deficient Knees

\begin{tabular}{lc}
\hline \multicolumn{1}{c}{ Tear zone } & Tears \\
\hline Posterior horn & $49(63.6)$ \\
Posterior horn-body & $21(27.3)$ \\
Posterior horn-anterior horn & $3(3.9)$ \\
Body & $4(5.2)$ \\
\hline
\end{tabular}

Values are presented as number (\%). 
Table 2. The Radial Zone Distribution of Lateral Meniscal Tears in Anterior Cruciate Ligament-deficient Knees

\begin{tabular}{lc}
\hline \multicolumn{1}{c}{ Tear zone } & Tears \\
\hline Posterior horn & $42(68.9)$ \\
Posterior horn-body & $4(6.6)$ \\
Posterior horn-anterior horn & $1(1.6)$ \\
Body & $10(16.4)$ \\
Body-anterior horn & $1(1.6)$ \\
Anterior horn & $3(4.9)$ \\
\hline
\end{tabular}

Values are presented as number (\%).

Table 3. The Circumferential Zone Distribution of Meniscal Tears in Anterior Cruciate Ligament-deficient Knees

\begin{tabular}{lc}
\hline Tear zone & Tears \\
\hline Peripheral & $99(71.7)$ \\
Central & $39(28.3)$ \\
\hline
\end{tabular}

Values are presented as number (\%).

differences were found with regard to age, sex, BMI, the interval from injury to ACL reconstruction, with the exception of PTS (Table 4). The incidence of medial meniscal tears was $22.2 \%$ (30/135) in the low group and 56.4\% (22/39) in the high group (Table 5). The difference in the incidence of medial meniscal tears was significant between the two groups $(\mathrm{p}<0.001)$.

\section{Discussion}

Prior studies have suggested several factors related to anterior tibial translation including axial loading, degree of knee flexion, and PTS ${ }^{13-15)}$.

Allen et al. ${ }^{5)}$ suggested that the meniscal wedge is the cause of medial meniscal tears in chronic ACL-deficient knees. On the other hand, a more mobile lateral meniscus is less likely to undergo shear stress in the ACL-deficient knees. The present study showed that the PTS was larger in the knees with medial meniscal tears than those without, but the slope was similar between the knees with lateral meniscal tears and those with intact meniscus.

Although the PTS was larger in patients with medial meniscal tears, this may not be clinically significant because of the relatively small difference in the mean PTS. However, when we analyzed using a different method, the difference between the low and high group was significant. Therefore, we concluded that a PTS $\geq 13^{\circ}$ is one of the risk factors for secondary medial meniscal tears in patients with ACL deficiency. Weiss et al. ${ }^{15)}$ reported that
Table 4. Patient Demographics

\begin{tabular}{lccc}
\hline \multicolumn{1}{c}{ Variable } & Low group & High group & p-value \\
\hline Number of patients & 135 & 39 & \\
Age (y) & $30.8 \pm 9.5$ & $30.5 \pm 9.3$ & 0.153 \\
Sex (male/female) & $115 / 20$ & $33 / 6$ & 0.116 \\
Body mass index & $24.1 \pm 2.5$ & $23.8 \pm 2.3$ & 0.185 \\
Time to surgery (mo) & $15.2 \pm 27.7$ & $16.2 \pm 27.7$ & 0.096 \\
\hline
\end{tabular}

Table 5. Difference of Number of Patients Who Have Meniscal Tears in Each Group

\begin{tabular}{lcrc}
\hline \multicolumn{1}{c}{ Site } & Low group & High group & p-value \\
\hline No tear & $57 / 135(42.2)$ & $4 / 39(10.3)$ & $<0.001$ \\
Medial meniscus & $30 / 135(22.2)$ & $22 / 39(56.4)$ & $<0.001$ \\
Lateral meniscus & $30 / 135(22.2)$ & $6 / 39(15.4)$ & 0.185 \\
Both meniscus & $18 / 135(13.3)$ & $7 / 39(17.9)$ & 0.153 \\
Any meniscus & $78 / 135(57.8)$ & $35 / 39(89.7)$ & $<0.001$ \\
\hline \multicolumn{2}{l}{ Values are presented as number (\%). }
\end{tabular}

acute stable peripheral meniscal tears with acute ACL injury have potential for healing. An accentuated anterior tibial shift in knees with increased tibial slope would focus a great deal of stress at the medial meniscal tear site and possibly prevent spontaneous healing of a meniscal tear. Thus, we suggest that PTS is one of the considerations for determining early ACL reconstruction to prevent secondary meniscal tears in patients with ACL injury and intact meniscus.

However, the present study has some limitations that require consideration. In order to know the exact time of meniscal injury, we need serial magnetic resonance (MR) images, including those taken immediately after trauma. Unfortunately, most patients do not have MR images taken immediately after a traumatic event. Also, other risk factors for secondary medial meniscal tears in ACL-deficient knees such as repetitive injury and patient activity level were not considered in this study.

\section{Conclusions}

The PTS was larger in patients with medial meniscal tears. A $\mathrm{PTS} \geq 13^{\circ}$ is one of the risk factors for secondary meniscal tears in chronic ACL-deficient knees. Therefore, we suggest that PTS is one of the considerations for determining early ACL reconstruction to prevent secondary meniscal tears in patients with ACL injury and intact meniscus. 


\section{References}

1. Church S, Keating JF. Reconstruction of the anterior cruciate ligament: timing of surgery and the incidence of meniscal tears and degenerative change. J Bone Joint Surg Br. 2005;87:1639-42.

2. Bellabarba C, Bush-Joseph CA, Bach BR Jr. Patterns of meniscal injury in the anterior cruciate-deficient knee: a review of the literature. Am J Orthop (Belle Mead NJ). 1997;26:18-23.

3. Thompson WO, Fu FH. The meniscus in the cruciatedeficient knee. Clin Sports Med. 1993;12:771-96.

4. Warren LF, Marshall JL. The supporting structures and layers on the medial side of the knee: an anatomical analysis. J Bone Joint Surg Am. 1979;61:56-62.

5. Allen CR, Wong EK, Livesay GA, Sakane M, Fu FH, Woo SL. Importance of the medial meniscus in the anterior cruciate ligament-deficient knee. J Orthop Res. 2000;18:10915.

6. Hsieh HH, Walker PS. Stabilizing mechanisms of the loaded and unloaded knee joint. J Bone Joint Surg Am. 1976;58:8793.

7. Indelicato PA, Bittar ES. A perspective of lesions associated with ACL insufficiency of the knee. A review of 100 cases. Clin Orthop Relat Res. 1985;(198):77-80.

8. Levy IM, Torzilli PA, Warren RF. The effect of medial meniscectomy on anterior-posterior motion of the knee. J Bone Joint Surg Am. 1982;64:883-8.

9. Sullivan D, Levy IM, Sheskier S, Torzilli PA, Warren RF. Medical restraints to anterior-posterior motion of the knee. J Bone Joint Surg Am. 1984;66:930-6.

10. Dejour H, Bonnin M. Tibial translation after anterior cruciate ligament rupture. Two radiological tests compared. J Bone Joint Surg Br. 1994;76:745-9.

11. Dejour H, Neyret P, Boileau P, Donell ST. Anterior cruciate reconstruction combined with valgus tibial osteotomy. Clin Orthop Relat Res. 1994;(299):220-8.

12. Brazier J, Migaud H, Gougeon F, Cotten A, Fontaine C, Duquennoy A. Evaluation of methods for radiographic measurement of the tibial slope. A study of 83 healthy knees. Rev Chir Orthop Reparatrice Appar Mot. 1996;82: 195-200.

13. Shoemaker SC, Markolf KL. The role of the meniscus in the anterior-posterior stability of the loaded anterior cruciatedeficient knee. Effects of partial versus total excision. J Bone Joint Surg Am. 1986;68:71-9.

14. Giffin JR, Vogrin TM, Zantop T, Woo SL, Harner CD. Effects of increasing tibial slope on the biomechanics of the knee. Am J Sports Med. 2004;32:376-82.

15. Weiss CB, Lundberg M, Hamberg P, DeHaven KE, Gillquist J. Non-operative treatment of meniscal tears. J Bone Joint Surg Am. 1989;71:811-22. 